International Journal of Trend in Scientific Research and Development (IJTSRD)

Volume: 3 | Issue: 3 | Mar-Apr 2019 Available Online: www.ijtsrd.com e-ISSN: 2456 - 6470

\title{
EHV Transmission Line Capacity Enhancement through Increase in SIL Level
}

\author{
Kuntal Verma1, Mratyunjay Sisodiya1, Dr. Babita Jain² \\ ${ }^{1}$ Student, ${ }^{2}$ Assistant Professor \\ 1,2Poornima College of Engineering, Jaipur, Rajasthan, India
}

\begin{abstract}
How to cite this paper: Kuntal Verma | Mratyunjay Sisodiya | Dr. Babita Jain "EHV Transmission Line Capacity Enhancement through Increase in SIL Level" Published in International Journal of Trend in Scientific Research and Development (ijtsrd), ISSN: 24566470, Volume-3 | Issue-3, April 2019, pp.604-606, URL: http://www.ijtsrd.co $\mathrm{m} /$ papers/ijtsrd228 68.pdf

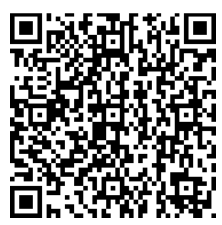
IITSRD22868
\end{abstract}

Copyright (c) 2019 by author(s) and International Journal of Trend in Scientific Research and Development Journal. This is an Open Access article distributed under the terms of the Creative Commons

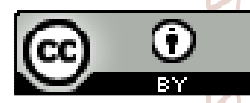
Attribution License (CC BY 4.0) (http://creativecommons.org/licenses/ by $/ 4.0$ )

\section{INTRODUCTION}

Power demand is continuously increasing every year and gap is being generated between power demand and supply. These power requirements can be met by adding new generating units or by expanding already existing system. There is a difference between power generation and demand in various regions, some are power surplus and some are deficient of power. Power can be utilized in a better manner by transferring it from power surplus to deficient region. Transferring power from power surplus to power deficient region requires long transmission lines and its EHV transmission lines should be used for long transmission lines as it has various advantages such as it increases transmission efficiency, reduce losses and limits the current. Hence for transmission lines EHV transmission lines should be used. Along with implementing EHV transmission lines their capacity should also be enhanced so that power can be utilized more effectively. Capacity of a transmission line is affected by its thermal and SIL limits. For long transmission lines only reason affecting capacity of transmission line is its SIL limit. So increasing SIL level should be considered for increasing capacity of EHV transmission lines, SIL level is decreases due to presence of large inductance and high surge impedance. To increase SIL level surge impedance and inductance of line should be decreased and this will improve capacity of EHV transmission line. Surge impedance loading and various factors affecting it are further discussed in detail.

\section{Theory of surge impedance loading}

SIL loading of a line is a loading at which total reactive power is balanced in a line, Total reactive power generated will be equal to total reactive power absorbed by line. If a line is loaded above its SIL then it will absorb volt ampere reactive from line and behaves as a shunt reactor and if it is loaded below its SIL it will supply volt ampere reactive to line and behaves as a shunt capacitor. At surge impedance loading

$$
\begin{aligned}
& \text { Capacitive VAR }=\text { inductive VAR } \\
& V^{2} / \mathrm{X}_{\mathrm{C}}=\mathrm{I}^{2} \mathrm{X}_{\mathrm{L}} \\
& \mathrm{V}^{2} / \mathrm{I}^{2}=\sqrt{(\mathrm{L} / \mathrm{C})} \\
& \mathrm{Z}_{\mathrm{C}}=\sqrt{(\mathrm{L} / \mathrm{C})}
\end{aligned}
$$

$\mathrm{Z}_{\mathrm{C}}$ is the surge impedance of line and surge impedance loading is given as follows-

$$
\mathrm{SIL}=\mathrm{V}^{2} / \mathrm{Z}_{\mathrm{c}}
$$

Ratings of generator and transformers are taken from SIL. It can be seen from above equation that SIL is inversely proportional to surge impedance. SIL can be improved by decreasing surge impedance of line or by decreasing inductance of line as $\mathrm{Z}_{\mathrm{C}}=\sqrt{ }(\mathrm{L} / \mathrm{C})$. inductance of line is given by 


$$
\mathrm{L}=\mathrm{L}_{\mathrm{S}}-\mathrm{L}_{\mathrm{M}}
$$

$\mathrm{L}_{\mathrm{S}=}$ self inductance of line $=2 * 10^{-7} \ln \left(1 / \mathrm{GMR}_{\mathrm{eq}}\right) \mathrm{H} / \mathrm{m}$

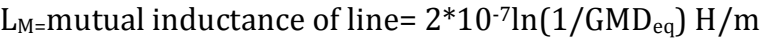

Here $\mathrm{GMR}_{\mathrm{eq}}$ is geometric mean radius of conductor and $\mathrm{GMD}_{\text {eq }}$ is geometric mean distance between conductors.

Inductance of line can be decreased by decreasing self inductance of line or by increasing mutual inductance of line and hence SIL can be improved. Self inductance can be decreased by increasing GMR (geometric mean radius) and mutual inductance can be increased by decreasing GMD (geometric mean distance).

At SIL of line sending end voltage will be equal to receiving end voltage and is equal to voltage at every point of line i.e. flat voltage profile is obtained at SIL.

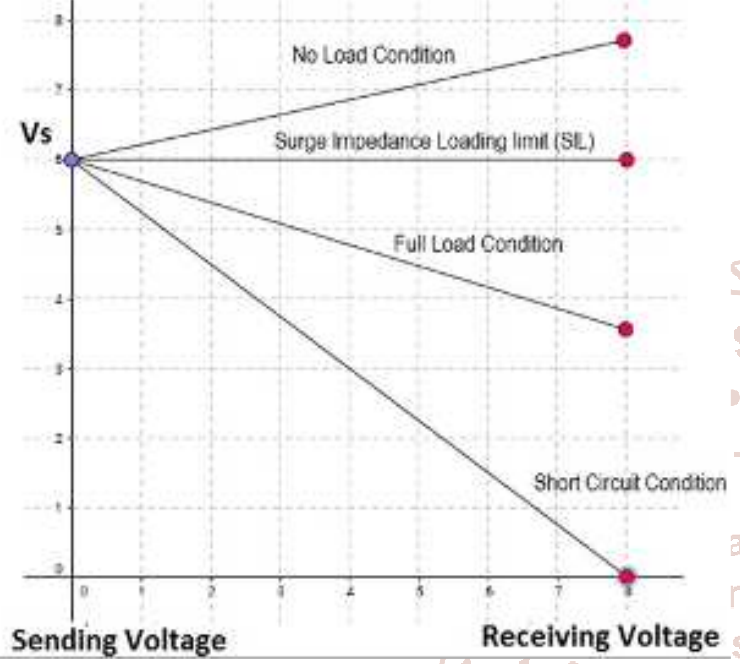

It can be seen in above graph that flat voltage profile is obtained at SIL. As shown in graph sending end and receiving end voltages are equal at surge impedance. SIL (surge impedance loading) is the loading of generators, transformers etc. at surge impedance. To enhance efficiency of transmission line SIL level of line should be improved.

\section{Methods of improving SIL}

Efficiency of a EHV transmission line can be enhanced by increased by increasing its surge impedance loading. Various methods of improving SIL are as follows-

\subsection{Decreasing phase to phase spacing}

SIL (surge impedance loading) level of a line can be improved by decreasing phase to phase spacing between the conductors of transmission line. On decreasing the value of phase to phase spacing between conductors, GMD (Geometric Mean Distance) will decrease, due to decrement in GMD mutual inductance of line will increase. Mutual inductance of line will increase and hence line inductance and surge impedance of line will decrease and this will result in increased SIL level. So to increase SIL phase to phase spacing should be decreased.

\subsection{Increasing bundle spacing}

SIL level of EHV transmission line can be improved by increasing bungle spacing. Increasing bundle spacing of a conductor will increase GMR (Geometric Mean Radius) of conductor. Self inductance of a line will decrease on increasing GMR. Decreased value of self inductance will lead to reduction in line inductance and surge impedance of transmission line and hence SIL level of a EHV Transmission line is improved.

\subsection{Increasing conductor diameter}

SIL level of EHV transmission line can be improved by increasing diameter of conductor. GMR (Geometric Mean Radius) of a conductor increases on increasing conductor diameter. Self inductance of EHV transmission line decreases on increasing GMR and hence line inductance and surge impedance of transmission line are reduced and SIL Level of transmission line is improved.

3.4 increasing number of sub-conductors per phase SIL level of EHV transmission line can be improved by increasing number of sub-conductors per phase. GMR (Geometric Mean Radius) of conductor will increase on increasing number of sub-conductors per phase. Self inductance of line decreases on increasing GMR and this reduces line inductance and surge impedance of transmission line. As surge impedance of transmission line is reduced SIL level of will increasing.

\subsection{Decreasing line to ground distance}

SIL level of a EHV transmission line can be improaved by reducing line to ground distance. If the effect of line to ground distance is considered, capacitance of a EHV transmission line increases on reducing line to ground distance. Increment in line capacitance leads to reduction in surge impedance of transmission line and hence SIL level of EHV transmission line is improved. But line to ground distance can't be decreased below a certain value due to safety reasons.

4. Comparison between different conductor and subconductor configurations for SIL

1. Horizontal configuration

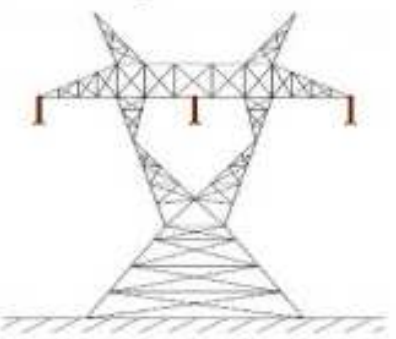

2. Delta Configuration

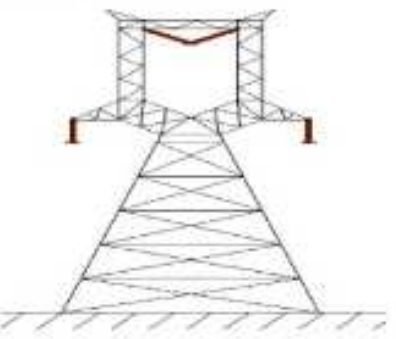

3. L Configuration

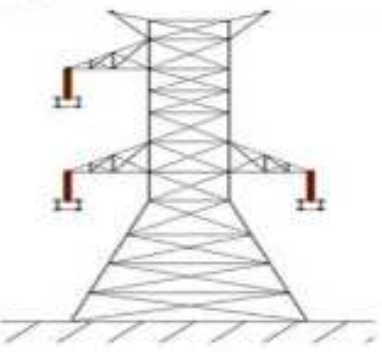


Different types of conductor configurations are used in the EHV transmission lines for the power transmission. These configurations are horizontal configuration, L-configuration and delta configuration. Configuration of conductors used in transmission line affects SIL level. SIL level is minimum for horizontal configuration, comparatively increased for L configuration and maximum for delta configuration. Right of way is maximum for horizontal configuration, comparatively decreased for delta configuration and minimum for Lconfiguration. For a $400 \mathrm{kV}$ line having 2 sub-conductors, $100 \mathrm{~cm}$ phase to phase spacing and $1000 \mathrm{~cm}$ line to ground distance SIL is increased $2.34 \%$ for L-configuration and $4.8 \%$ increased for delta configuration. If the effect of line to ground distance is considered SIL is $1.35 \%$ increased for Lconfiguration and $3.63 \%$ increased for delta configuration. Effect of conductor configurations on SIL is shown in the following table_

\begin{tabular}{|c|c|c|c|}
\hline Configuration & horizontal & delta & L-configuration \\
\hline SIL(MW) & 526.7 & 551.9 & 539.0 \\
\hline SILg(MW) & 537.0 & 556.5 & 544.27 \\
\hline
\end{tabular}

Configuration of sub-conductors in a line conductor also affects SIL level. For a conductor having four sub-conductors per phase square trapezoidal and rectangular configurations are used. SIL is minimum for square configuration, comparatively increased for trapezoidal configuration and maximum for rectangular configuration. Area is maximum for square configuration, comparatively reduced for rectangular configuration and much reduction in area is obtained for trapezoidal configuration hence material required and cost is minimum for trapezoidal configuration.

\section{Advantages and issues associated with high SIL lines Advantages-}

There are various advantages associated with high SIL lines due to which these lines are being implemented. Some of the advantages are enhanced power transfer capability, improved stability, reduced losses and better voltage regulation. Along with these reduced environmental impacts, optimal utilization of right of way and optimum transmission cost are obtained.

Issues-

Along with advantages, high SIL transmission lines have several issues associated with it. these lines generate more MVAR in comparison of conventional lines, therefore requires power management studies. Special hardware and accessories are required in implementation of high SIL lines. There are also some problems arise due to the electric fields of these lines.

\section{Conclusion}

EHV transmission lines transmit large amount of power over large distance and due to various advantages such as increased efficiency, reduced losses etc. EHV transmission lines are being expanded. To meet various requirements like transmitting power from one region to another region, to meets additional power demand and for various another purposes transmission system is needed to be expanded and improved, this necessitates the EHV transmission lines. Capacity enhancement of these EHV transmission lines is needed to be considered, and for this purpose SIL level of line is needed to be improved. SIL can be improved by using various techniques discussed in paper. Increased SIL level means increased power transmission capacity of EHV transmission lines. Increasing SIL level provides various advantages but there are certain issues associated with the high value of SIL which are needed to be considered. By balancing issues and keeping all the factors in consideration it can be concluded that SIL level of EHV transmission lines should be increased to enhance the capacity of EHV transmission lines.

\section{References}

[1] research paper on SIL level enhancement of $765 \mathrm{kv}$ long line through bundle configuration by jitendra jamnani and varun patel published in 2016

[2] research paper on techniques to increase SIL of AC transmission line by varun patel and J.G. jamnani published in 2015

[3] https://www.electricalindia.in

[4] www.gmail.com

[5] www.youtube.com

[6] https://sci.hub.tw

[7] https://ieeexplore.ieee.org

[8] www.wikipidea.org

[9] https://electrical4u.com

[10] https://semanticscholar.org

[11] Research paper on EHV line capacity enhancement through increase in SIL by R.N. Nayak and Y.K. sehgal. 